REVISTA DE LA

UNIÓN MATEMÁTICA ARGENTINA

Vol. 59, No. 2, 2018, Pages 265-284

Published online: February 20, 2018

\title{
A TOPOLOGICAL DUALITY FOR MILDLY DISTRIBUTIVE MEET-SEMILATTICES
}

\author{
SERGIO A. CELANI AND LUCIANO J. GONZÁLEZ
}

\begin{abstract}
We develop a topological duality for the category of mildly distributive meet-semilattices with a top element and certain morphisms between them. Then, we use this duality to characterize topologically the lattices of Frink ideals and filters, and we also obtain a topological representation for some congruences on mildly distributive meet-semilattices.
\end{abstract}

\section{INTRODUCTION}

Duality theory for ordered algebraic structures goes back to Stone's pioneering work [15. He proved that the category of Boolean algebras and Boolean homomorphisms is dually equivalent to the category of Boolean spaces (compact and totally disconnected spaces) and continuous maps. This duality was generalized to distributive lattices by Stone himself [16]. He showed a duality between the category of distributive lattices and lattice homomorphisms and the category of spectral spaces (sober spaces in which the compact open sets form a base that is closed under finite intersections) and spectral functions (functions whose inverse image sends compact open sets to compact open sets).

A different approach was used by Priestley [14] to obtain a topological duality for distributive lattices in term of ordered Hausdorff topological spaces, later known as Priestley spaces.

These two approaches have been followed to obtain topological dualities for several ordered algebraic structures. In particular, for distributive meet-semilattices [5, 2], for implicative meet-semilattices [1], for distributive nearlattices [3] and also in a more general setting: For a class of partially ordered sets satisfying a distributivity condition [7, 10].

2010 Mathematics Subject Classification. Primary 06A12; Secondary 18B30, 06B10.

Key words and phrases. Semilattices, distributivity on semilattices, duality theory, congruences.

This research was supported by Consejo Nacional de Investigaciones Científicas y Técnicas (Argentina) under the grant PIP 112-20150-100412CO. The first author was also partially supported by the EU H2020-MSCARISE-2015 project 689176-SYSMICS. The second author was also partially supported by Universidad Nacional de La Pampa (Fac. de Cs. Exactas y Naturales) under the grant P.I. 64 M, Res. 432/14 CD. 
We focus in this paper on mildly distributive meet-semilattices with a top element. The mild distributivity condition was introduced and studied by Hickman [12. He paid particular attention to the study of morphisms and congruences on these structures. Recently, we have continued the algebraic study of mildly distributive meet-semilattices in [4] we obtained new characterizations of the mildly distributivity condition, and we studied the collections of Frink ideals and filters.

Now, in the present article, we develop a dual equivalence between the category of mildly distributive meet-semilattices and strong homomorphisms and the category of certain topological spaces and particular continuous maps. We follow the topological approach of Stone to achieve our aim. Then, we use this topological duality to obtain topological representations for the lattices of filters and Frink ideals of mildly distributive meet-semilattices and finally, we get a topological representation for some congruences.

The paper is organized as follows. In Section 2, we introduce some basic facts needed for what follows. In Section 3 , we present the definition of mildly distributivity on semilattices, and we show some known results in the literature. We also introduce the morphisms between mildly distributive semilattices, called strong homomorphisms, which are important for the aim of the paper. We show in Section 4 a set-theoretic representation for mildly distributive semilattices. Then, in Section 5, we present the definition of the md-spaces and we develop a categorical dual equivalence between the mildly distributive semilattices and the md-spaces. Section 6 is devoted to obtain topological representations of the lattices of Frink ideals and filters. We show that the Frink ideals of a mildly distributive semilattice correspond to some particulars subsets of its dual md-space and the filters are in correspondence with a subclass of closed subsets. Lastly, in Section 7, we show a correspondence between some congruences on a mildly distributive semilattice and some subspaces of its dual md-space.

\section{Preliminaries}

In this section, we introduce the main notions and results for what follows in the paper. We assume that the reader is familiar with elementary order theoretical concepts (see [6]).

Let $f: X \rightarrow Y$ be a function. Let $A \subseteq X$ and $B \subseteq Y$. We denote the image of the subset $A$ by $f[A]=\{f(a): a \in A\}$ and the inverse image of the subset $B$ by $f^{-1}[B]=\{x \in X: f(x) \in B\}$. By $f^{-1}(): \mathcal{P}(Y) \rightarrow \mathcal{P}(X)$ we denote the inverse image function, that maps every set $B \subseteq Y$ to $f^{-1}(B)=f^{-1}[B]$.

Let $P=\langle P, \leq\rangle$ be a poset. A subset $Y \subseteq P$ is a downset of $P$ provided that for every $b \in P$ if $b \leq a$ for some $a \in Y$, then $b \in Y$. Dually, it is an upset if for every $b \in P$, if $a \leq b$ for some $a \in Y$, then $b \in Y$. For $a \in P,(a]$ denotes the downset set $\{b \in P: b \leq a\}$ and $[a)$ denotes the upset $\{b \in P: a \leq b\}$. If $Y \subseteq P$, let $Y^{u}:=\{a \in P:(\forall y \in Y)(y \leq a)\}$ the set of all upper bounds of $Y$ and $Y^{l}:=\{a \in P:(\forall y \in Y)(a \leq y)\}$ the set of all lower bounds of $Y$. Note that if $a \in P$, then $(a]=\{a\}^{u l}$ and $[a)=\{a\}^{l u}$. 
A meet-semilattice is an algebra $L=\langle L, \wedge\rangle$ of type (2) such that the operation $\wedge$ is idempotent, commutative and associative. As usual, a partial order $\leq$ is defined by $a \leq b$ if and only if $a \wedge b=a$. A meet-semilattice with top element is an algebra $\langle L, \wedge, 1\rangle$ of type $(2,0)$ such that $\langle L, \wedge\rangle$ is a meet-semilattice and $a \wedge 1=a$ for all $a \in L$ and a bounded meet-semilattice is an algebra $\langle L, \wedge, 0,1\rangle$ of type $(2,0,0)$ such that $\langle L, \wedge, 1\rangle$ is a meet-semilattice with top element and $a \wedge 0=0$ for all $a \in L$.

Let $L$ be a meet-semilattice. A nonempty subset $F \subseteq L$ is said to be a filter of $L$ if (i) it is an upset of $L$ and (ii) $a, b \in F$ implies $a \wedge b \in F$. We denote the collection of all filters of $L$ by $\mathrm{Fi}(L)$. It is easy to check that an arbitrary intersection of filters of $L$ is either the empty set or a filter. Thus, for a nonempty subset $X$ of $L$, there exists the least filter of $L$ that contains $X$; we denote this filter by $\operatorname{Fig}(X)$. A filter $F \in \mathrm{Fi}(L)$ is proper if $F \neq L$. A proper filter $F$ of $L$ is called meet-prime when for all filters $F_{1}, F_{2}$ of $L, F_{1} \cap F_{2} \subseteq F$ implies $F_{1} \subseteq F$ or $F_{2} \subseteq F$. We denote by $\mathrm{Fi}_{\text {mpr }}(L)$ the family of all meet-prime filters of $L$.

Since in a meet-semilattice the join of two elements may not exist, the notion of ideal, which generalizes the usual notion of ideal for lattices, has several possible definitions. Here we need two of them. Let $L$ be a meet-semilattice. A subset $I \subseteq L$ is said to be a Frink ideal of $L$ if for every finite $X \subseteq I, X^{u l} \subseteq I$ (cf. [9]). Let us denote by $\operatorname{Fld}(L)$ the collection of all Frink ideals of $L$. It should be noted that the empty set may be a Frink ideal, this depend on whether or not the meetsemilattice has bottom element. It is clear that Frink ideals are downsets. It is easy to show that a subset $I \subseteq L$ is a Frink ideal if and only if for every finite $A \subseteq I$ and $c \in L, \bigcap_{a \in A}[a) \subseteq[c$ ) implies $c \in I$; moreover if $L$ has bottom element, then in the previous characterization of Frink ideals is enough to consider only nonempty finite subsets $A \subseteq I$.

It is known that $\operatorname{Fld}(L)$ is an algebraic closure system and its associated closure operator is defined by: For $X \subseteq L$,

$$
\langle X\rangle=\left\{a \in L: \bigcap_{x \in X_{0}}[x) \subseteq[a) \text { for some finite } X_{0} \subseteq X\right\} .
$$

Hence, we have that $\operatorname{FId}(L)$ is a complete lattice with respect to the inclusion order. On the other hand, a nonempty subset $I \subseteq L$ is said to be an order ideal of $L$ if (i) it is a downset of $L$ and (ii) for every $a, b \in I$ there is $c \in I$ such that $a, b \leq c$. It is easily checked that every order ideal is a Frink ideal.

Lemma 2.1. Let $L$ be a meet-semilattice. Let $F$ be a proper filter of $L$. Then, $F$ is meet-prime if and only if $L \backslash F$ is an order ideal of $L$.

There is another possible notion of "prime" filter in the framework of meetsemilattices, which will be fundamental to develop our topological duality for the category of mildly distributive meet-semilattices. The notion of an optimal filter has been considered by Bezhanishvili and Jansana [1, 2, among others.

Definition 2.2. Let $L$ be a meet-semilattice. A proper filter $F$ of $L$ is called optimal if $L \backslash F$ is a Frink ideal. We denote by $\operatorname{Opt}(L)$ the collection of all optimal filters of $L$. 
It should be noted that a filter $P$ of a bounded meet-semilattice $L$ is optimal if and only if for all $a_{1}, \ldots, a_{n}, a \in L$, if $a_{1}, \ldots, a_{n} \notin P$ and $\left[a_{1}\right) \cap \cdots \cap\left[a_{n}\right) \subseteq[a)$, then $a \notin P$.

Now we present some topological notions needed in the article. We assume that the reader is familiar with elementary topological concepts. Our primary reference for general topology is 8 .

Let $\langle X, \mathcal{T}\rangle$ be a topological space. Let us denote by $\mathcal{C}(X)$ the collection of all closed subsets of $X$. For a subset $Y \subset X, \operatorname{cl}(Y)$ denotes the topological closure of $Y$, that is, $\operatorname{cl}(Y)=\bigcap\{A \in \mathcal{C}(X): Y \subseteq A\}$. For $x \in X$, we write $\operatorname{cl}(x)$ instead of $\operatorname{cl}(\{x\})$.

A closed subset $A$ of a topological space $X$ is called irreducible when for all closed subsets $B$ and $C$ of $X$, if $A \subseteq B \cup C$, then $A \subseteq B$ or $A \subseteq C$. A topological space $X$ is said to be sober when for every irreducible closed subset $A$ of $X$ there exists a unique element $x \in X$ such that $A=\operatorname{cl}(x)$. For more information about sober spaces, we refer the reader to [13].

\section{Mildly Distributive MeET-Semilattices}

The algebraic concepts on mildly distributive meet-semilattices presented in this section are due to Hickman [12] and due to us [4]; we direct the reader to these references for more details.

Definition 3.1. A mildly distributive meet-semilattice (md-semilattice for short) is a meet-semilattice $L=\langle L, \wedge\rangle$ such that the lattice $\operatorname{FId}(L)$ is a distributive lattice.

Theorem $3.2([12])$. Let $L$ be a meet-semilattice. Then, $L$ is mildly distributive if and only if for all $a_{1}, \ldots, a_{n} \in L$ and $a \in L$, if $\left[a_{1}\right) \cap \cdots \cap\left[a_{n}\right) \subseteq[a)$, then $a=\left(a \wedge a_{1}\right) \vee\left(a \wedge a_{2}\right) \vee \cdots \vee\left(a \wedge a_{n}\right)$.

Theorem 3.3 ([4). Let $L$ be an md-semilattice. Let $F$ be a filter and $I$ a Frink ideal of $L$. If $F \cap I=\emptyset$, then there exists an optimal filter $P$ of $L$ such that $F \subseteq P$ and $I \cap P=\emptyset$.

Proof. It is a consequence of Zorn's lemma.

The following corollary will be useful in what follows.

Corollary 3.4. Let $L$ be an md-semilattice.

(1) If $F$ is a filter of $L$ and $a \in L$ is such that $a \notin F$, then there exists an optimal filter $P$ of $L$ such that $F \subseteq P$ and $a \notin P$.

(2) If $a \not \leq b$, then there exists $P \in \operatorname{Opt}(L)$ such that $a \in P$ and $b \notin P$.

Let $L$ and $M$ be meet-semilattices. A map $h: L \rightarrow M$ is said to be a homomorphism if for all $a, b \in L$ we have that $h(a \wedge b)=h(a) \wedge h(b)$. A homomorphism $h: L \rightarrow M$ is called a join-homomorphism f $^{\text {if }} h$ preserves all existing finite joins, that is, if $a_{1}, \ldots, a_{n} \in L$ and $a_{1} \vee \cdots \vee a_{n}$ there exists in $L$, then $h\left(a_{1}\right) \vee \cdots \vee h\left(a_{n}\right)$ there exists in $M$ and equal to $h\left(a_{1} \vee \cdots \vee a_{n}\right)$. We say that a map $h: L \rightarrow M$

\footnotetext{
*In [12, the join-homomorphisms are called join partial homomorphisms.
} 
is a strong homomorphism (see [12] and [2]) if it is a homomorphism and for all $a_{1}, \ldots, a_{n}, a \in L$,

$$
\left[a_{1}\right) \cap \cdots \cap\left[a_{n}\right) \subseteq[a) \Longrightarrow\left[h\left(a_{1}\right)\right) \cap \cdots \cap\left[h\left(a_{n}\right)\right) \subseteq[h(a))
$$

holds.

Let $L$ and $M$ be meet-semilattices. If $h: L \rightarrow M$ is a strong homomorphism, then $h$ is a join-homomorphism and the converse is not always true. But, if $L$ is an md-semilattice and $M$ is an arbitrary meet-semilattice, then a map $h: L \rightarrow$ $M$ is a strong homomorphism iff it is a join-homomorphism (this can be seen in [12]). Thus, strong homomorphisms and join-homomorphisms coincide for mdsemilattices. Now, we present another characterization of strong homomorphisms between md-semilattices.

Proposition 3.5 ([4]). Let $L$ and $M$ be md-semilattices having top elements and let $h: L \rightarrow M$ be an order-preserving map that preserves top elements. Then, the following conditions are equivalent:

(1) $h$ is a strong homomorphism;

(2) for every $Q \in \operatorname{Opt}(M), h^{-1}(Q) \in \operatorname{Opt}(L) \cup\{L\}$.

\section{RePRESEntation theOREM FOR MD-SEMilattices}

Let $\langle L, \wedge, 1\rangle$ be an md-semilattice with top element 1 . All the md-semilattices considered in the rest of the paper will have top element.

Recall that $\operatorname{Opt}(L)$ denotes the collection of all optimal filters of $L$. Let us consider the set $\mathrm{X}(L):=\operatorname{Opt}(L) \cup\{L\}$ and the following map $\varphi_{L}: L \rightarrow \mathcal{P}(\mathrm{X}(L))$ defined by

$$
\varphi_{L}(a)=\{P \in \mathrm{X}(L): a \in P\}
$$

for each $a \in L$. Let us consider the following families:

$$
\mathcal{D}(\mathrm{X}(L)):=\varphi_{L}[L]=\left\{\varphi_{L}(a): a \in L\right\} \quad \text { and } \quad \mathcal{K}_{L}:=\left\{\varphi_{L}(a)^{c}: a \in L\right\},
$$

where for every $a \in L, \varphi_{L}(a)^{c}=\mathrm{X}(L) \backslash \varphi_{L}(a)=\{P \in \mathrm{X}(L): a \notin P\}$.

Notice that for every $a, b \in L$, we have $\varphi_{L}(a) \cap \varphi_{L}(b)=\varphi_{L}(a \wedge b)$ and $\varphi_{L}(1)=$ $\mathrm{X}(L)$. Then, $\langle\mathcal{D}(\mathrm{X}(L)), \cap, \mathrm{X}(L)\rangle$ is a meet-semilattice with top element. As a consequence of Corollary 3.4 we have the following result:

Proposition 4.1. The map $\varphi_{L}: L \rightarrow \mathcal{D}(\mathrm{X}(L))$ is an isomorphism of meet-semilattices with top element.

Since $\varphi_{L}$ is an isomorphism, it follows that $\varphi_{L}$ preserves all existing finite joins of $L$. Moreover, if $a, b \in L$ are such that $a \vee b$ exists in $L$, then

$$
\varphi_{L}(a \vee b)=\varphi_{L}(a) \cup \varphi_{L}(b) .
$$

For every md-semilattice $L$ we define the structure

$$
\mathrm{X}(L)=\left\langle\mathrm{X}(L), \mathcal{T}_{L}, \mathcal{K}_{L}\right\rangle
$$

where $\mathcal{T}_{L}$ is a topology on $\mathrm{X}(L)$ generated by the family $\mathcal{K}_{L}$. That is, $\mathcal{T}_{L}$ is the collection of all unions of finite intersections of members of $\mathcal{K}_{L}$. In other words, $\mathcal{K}_{L}$ is a subbase for a topology $\mathcal{T}_{L}$ on $\mathrm{X}(L)$. 
Proposition 4.2. Let $L$ be an md-semilattice. Then,

(1) the subbase $\mathcal{K}_{L}$ is closed under finite unions and $\emptyset \in \mathcal{K}_{L}$;

(2) $\left\langle\mathrm{X}(L), \mathcal{T}_{L}\right\rangle$ is $T_{0}$-space;

(3) $\bigcap_{a \in L} \varphi_{L}(a)=\{L\}$;

(4) for every $a \in L, \varphi_{L}(a)^{c}$ is a compact open subset of the space $\mathrm{X}(L)$;

(5) for all nonempty subsets $A$ and $B$ of $L$ such that

$$
\bigcap\left\{\varphi_{L}(a): a \in A\right\} \subseteq \bigcup\left\{\varphi_{L}(b): b \in B\right\},
$$

there exist $\left\{a_{1}, \ldots, a_{n}\right\} \subseteq A$ and $\left\{b_{1}, \ldots, b_{m}\right\} \subseteq B$ such that

$$
\varphi_{L}\left(a_{1}\right) \cap \cdots \cap \varphi_{L}\left(a_{n}\right) \subseteq \varphi_{L}\left(b_{1}\right) \cup \cdots \cup \varphi_{L}\left(b_{m}\right) .
$$

Proof. Properties (1)-(3) are immediate consequences of the definition of the map $\varphi_{L}$ and the construction of the structure $\left\langle\mathrm{X}(L), \mathcal{T}_{L}, \mathcal{K}_{L}\right\rangle$.

(4) Let $a \in L$. In order to prove that $\varphi_{L}(a)^{c}$ is compact we use that $\mathcal{K}_{L}$ is a subbase for $\mathrm{X}(L)$. So, let $\left\{a_{i}: i \in I\right\} \subseteq L$ be such that $\varphi_{L}(a)^{c} \subseteq \bigcup_{i \in I} \varphi_{L}\left(a_{i}\right)^{c}$. Thus, $\bigcap_{i \in I} \varphi_{L}\left(a_{i}\right) \subseteq \varphi_{L}(a)$. We consider the filter $F:=\operatorname{Fig}\left(\left\{a_{i}: i \in I\right\}\right)$. If $a \notin F$, then by Corollary 3.4 there is an optimal filter $P$ such that $F \subseteq P$ and $a \notin P$. So, $P \in \bigcap_{i \in I} \varphi_{L}\left(a_{i}\right)$ and $P \notin \varphi_{L}(a)$, which is a contradiction. Thus, we have $a \in F$. Then, there exist $i_{1}, \ldots, i_{n} \in I$ such that $a_{i_{1}} \wedge \cdots \wedge a_{i_{n}} \leq a$. Now, by Proposition 4.1 we obtain $\varphi_{L}\left(a_{i_{1}}\right) \cap \cdots \cap \varphi_{L}\left(a_{i_{n}}\right) \subseteq \varphi_{L}(a)$. Hence, $\varphi_{L}(a)^{c} \subseteq \varphi_{L}\left(a_{i_{1}}\right)^{c} \cup \cdots \cup \varphi_{L}\left(a_{i_{n}}\right)^{c}$ and therefore this implies that $\varphi_{L}(a)^{c}$ is compact.

(5) Let $A, B \subseteq L$ be such that

$$
\bigcap\left\{\varphi_{L}(a): a \in A\right\} \subseteq \bigcup\left\{\varphi_{L}(b): b \in B\right\} .
$$

Let $F$ be the filter generated by $A$ and let $I$ be the Frink ideal generated by $B$. Then $F \cap I \neq \emptyset$. Otherwise, by Theorem 3.3 there exists $P \in \operatorname{Opt}(L)$ such that $F \subseteq P$ and $I \cap P=\emptyset$. So, $P \in \bigcap\left\{\varphi_{L}(a): a \in A\right\}$ and $P \notin \bigcup\left\{\varphi_{L}(b): b \in B\right\}$, which is impossible. Now, since $F \cap I \neq \emptyset$, there exist $\left\{a_{1}, \ldots, a_{n}\right\} \subseteq A$ and $\left\{b_{1}, \ldots, b_{m}\right\} \subseteq B$ such that $\left[b_{1}\right) \cap \cdots \cap\left[b_{m}\right) \subseteq\left[a_{1} \wedge \cdots \wedge a_{n}\right)$. Hence, we obtain that $\varphi_{L}\left(a_{1}\right) \cap \cdots \cap \varphi_{L}\left(a_{n}\right) \subseteq \varphi_{L}\left(b_{1}\right) \cup \cdots \cup \varphi_{L}\left(b_{m}\right)$.

Remark 4.3. By Proposition 4.1, we have

$$
\begin{aligned}
{\left[a_{1}\right) \cap \cdots \cap\left[a_{n}\right) \subseteq[a) } & \Longleftrightarrow a \in\left\{a_{1}, \ldots, a_{n}\right\}^{u l} \\
& \Longleftrightarrow \varphi_{L}(a) \in\left\{\varphi_{L}\left(a_{1}\right), \ldots, \varphi_{L}\left(a_{n}\right)\right\}^{u l} \\
& \Longleftrightarrow\left[\varphi_{L}\left(a_{1}\right)\right) \cap \cdots \cap\left[\varphi_{L}\left(a_{n}\right)\right) \subseteq\left[\varphi_{L}(a)\right)
\end{aligned}
$$

for all $a_{1}, \ldots, a_{n}, a \in L$.

Proposition 4.4. Let $L$ be an $m d$-semilattice and let $a_{1}, \ldots, a_{n}, a \in L$. Then,

$$
\left[\varphi_{L}\left(a_{1}\right)\right) \cap \cdots \cap\left[\varphi_{L}\left(a_{n}\right)\right) \subseteq\left[\varphi_{L}(a)\right) \Longleftrightarrow \varphi_{L}(a) \subseteq \varphi_{L}\left(a_{1}\right) \cup \cdots \cup \varphi_{L}\left(a_{n}\right) .
$$

Proof. $\Rightarrow)$ Assume that $\left[\varphi_{L}\left(a_{1}\right)\right) \cap \cdots \cap\left[\varphi_{L}\left(a_{n}\right)\right) \subseteq\left[\varphi_{L}(a)\right)$ and suppose that $P \in \varphi_{L}(a)$ and $P \notin \varphi_{L}\left(a_{1}\right) \cup \cdots \cup \varphi_{L}\left(a_{n}\right)$. So, $a \in P$ and $a_{1}, \ldots, a_{n} \notin P$. By the previous remark we have that $\left[a_{1}\right) \cap \cdots \cap\left[a_{n}\right) \subseteq[a$ ). Since $P$ is optimal, it follows that $a \in L \backslash P$, which is a contradiction. 
$\Leftarrow)$ Reciprocally, assume that $\varphi_{L}(a) \subseteq \varphi_{L}\left(a_{1}\right) \cup \cdots \cup \varphi_{L}\left(a_{n}\right)$. Let $\varphi_{L}(x) \in$ $\left[\varphi_{L}\left(a_{1}\right)\right) \cap \cdots \cap\left[\varphi_{L}\left(a_{n}\right)\right)$. So, it is clear that $\varphi_{L}\left(a_{1}\right) \cup \cdots \cup \varphi_{L}\left(a_{n}\right) \subseteq \varphi_{L}(x)$. Then, $\varphi_{L}(x) \in\left[\varphi_{L}(a)\right)$.

Remark 4.5. Let $L$ be a bounded distributive lattice. It is straightforward to check directly that the collections of meet-prime filters and optimal filters coincide and Frink ideals are exactly the ideals in lattice. Thus, if we consider $X(L)^{*}:=X(L) \backslash\{L\}=\operatorname{Opt}(L)$ instead of $X(L)$ for the topological construction of this section, then we obtain the classical representation theorem for bounded distributive lattices due to Stone [16]; see also [11, II-5].

\section{A DUALity FOR MD-SEMILATtices}

We will say that a structure $\langle X, \mathcal{T}, \mathcal{K}\rangle$ is a topological space with a subbase $\mathcal{K}$ if $\langle X, \mathcal{T}\rangle$ is a topological space and $\mathcal{K}$ is a distinguished subbase of the topology $\mathcal{T}$. For every topological space $\langle X, \mathcal{T}, \mathcal{K}\rangle$ with a subbase $\mathcal{K}$, we consider the set

$$
\mathcal{D}_{\mathcal{K}}(X)=\left\{A \subseteq X: A^{c} \in \mathcal{K}\right\}
$$

and the poset $\left\langle\mathcal{D}_{\mathcal{K}}(X), \subseteq\right\rangle$. For brevity, and when there is no danger of confusion, we omit the subscript $\mathcal{K}$.

Definition 5.1. An $m d$-space is a topological space $\langle X, \mathcal{T}, \mathcal{K}\rangle$ with a subbase $\mathcal{K}$ such that:

(1) $\mathcal{K}$ is a family of compact and open subsets of the topology $\mathcal{T}$ closed under finite unions and $\emptyset \in \mathcal{K}$;

(2) $\langle X, \mathcal{T}\rangle$ is a $T_{0}$-space;

(3) $\bigcap\{A: A \in \mathcal{D}(X)\} \neq \emptyset$;

(4) for every subset $\left\{A_{1}, \ldots, A_{n}, A\right\} \subseteq \mathcal{D}(X)$,

$$
\left[A_{1}\right) \cap \cdots \cap\left[A_{n}\right) \subseteq[A) \Longleftrightarrow A \subseteq A_{1} \cup \cdots \cup A_{n} ;
$$

(5) for all nonempty families $\mathcal{A}$ and $\mathcal{B}$ of elements of $\mathcal{D}(X)$, if $\bigcap \mathcal{A} \subseteq \cup \mathcal{B}$ then there exist $\left\{A_{1}, \ldots, A_{n}\right\} \subseteq \mathcal{A}$ and $\left\{B_{1}, \ldots, B_{m}\right\} \subseteq \mathcal{B}$ such that

$$
A_{1} \cap \cdots \cap A_{n} \subseteq B_{1} \cup \cdots \cup B_{m} .
$$

For simplicity, we will sometimes say that $X$ is an md-space, understanding that there is a distinguished subbase $\mathcal{K}$ defining the topology $\mathcal{T}$ corresponding to space $X$.

By condition (1) of the definition of md-spaces, it follows that for every md-space $\langle X, \mathcal{T}, \mathcal{K}\rangle,\langle\mathcal{D}(X), \cap, X\rangle$ is a meet-semilattice with top element.

Proposition 5.2. Let $X$ be an md-space. Then, the meet-semilattice $\mathcal{D}(X)$ is mildly distributive.

Proof. It is a consequence of condition (4) of Definition 5.1 and Theorem 3.2

Proposition 5.3. Let $L$ be an md-semilattice. Then, $\left\langle\mathrm{X}(L), \mathcal{T}_{L}, \mathcal{K}_{L}\right\rangle$ is an $m d$ space. 
Proof. It follows from the definition of $\left\langle\mathrm{X}(L), \mathcal{T}_{L}, \mathcal{K}_{L}\right\rangle$ and by Propositions 4.2 and 4.4

For every md-semilattice $L,\left\langle\mathrm{X}(L), \mathcal{T}_{L}, \mathcal{K}_{L}\right\rangle$ is called the dual md-space of $L$. Now, the following corollary is an immediate consequence from the previous proposition and Proposition 4.1

Corollary 5.4. For every $m d$-semilattice $L$, there exists an $m d$-space $\langle X, \mathcal{T}, \mathcal{K}\rangle$ such that $L$ is isomorphic to the md-semilattice $\mathcal{D}(X)$.

Let $\langle X, \mathcal{T}, \mathcal{K}\rangle$ be an md-space. We define the map $H_{X}: X \rightarrow \mathrm{X}(\mathcal{D}(X))$ by

$$
H_{X}(x)=\{A \in \mathcal{D}(X): x \in A\}
$$

for each $x \in X$. Let us see that $H_{X}$ is well defined. Let $x \in X$. It is clear that $H_{X}(x)$ is a filter of the md-semilattice $\mathcal{D}(X)$. If $H_{X}(x)=\mathcal{D}(X)$, then $H_{X}(x) \in$ $\mathrm{X}(\mathcal{D}(X))$. Assume that $H_{X}(x) \neq \mathcal{D}(X)$. Now, in order to show that $H_{X}(x)$ is optimal, let $A_{1}, \ldots, A_{n} \notin H_{X}(x)$ and let $A \in \mathcal{D}(X)$ be such that $\left[A_{1}\right) \cap \cdots \cap\left[A_{n}\right) \subseteq$ $[A)$. So, $x \notin A_{1} \cup \cdots \cup A_{n}$ and $A \subseteq A_{1} \cup \cdots \cup A_{n}$. Then, $x \notin A$ and thus $A \notin H_{X}(x)$. Therefore, $H_{X}(x) \in \mathrm{X}(\mathcal{D}(X))$.

Theorem 5.5. Let $\langle X, \mathcal{T}, \mathcal{K}\rangle$ be a topological space satisfying conditions (1)-(4) of Definition 5.1. Then, the following conditions are equivalent:

(1) For all nonempty families $\mathcal{A}$ and $\mathcal{B}$ of elements of $\mathcal{D}(X)$, if $\bigcap \mathcal{A} \subseteq \cup \mathcal{B}$ then there exist $\left\{A_{1}, \ldots, A_{n}\right\} \subseteq \mathcal{A}$ and $\left\{B_{1}, \ldots, B_{m}\right\} \subseteq \mathcal{B}$ such that

$$
A_{1} \cap \cdots \cap A_{n} \subseteq B_{1} \cup \cdots \cup B_{m} .
$$

(2) The map $H_{X}: X \rightarrow \mathrm{X}(\mathcal{D}(X))$ is onto.

Proof. (1) $\Rightarrow(2)$ Let $P \in \mathrm{X}(\mathcal{D}(X))$. Suppose that $P=\mathcal{D}(X)$. By condition (3) of Definition 5.1, there exists $x \in X$ such that $H_{X}(x)=\mathcal{D}(X)=P$. Thus, we assume that $P \in \operatorname{Opt}(\mathcal{D}(X))$. We prove that

$$
\bigcap\{A: A \in P\} \cap \bigcap\left\{B^{c}: B \notin P\right\} \neq \emptyset \text {. }
$$

Suppose towards a contradiction that $\bigcap\{A: A \in P\} \subseteq \bigcup\{B: B \notin P\}$. Thus, there exist finite families $\left\{A_{1}, \ldots, A_{n}\right\} \subseteq P$ and $\left\{B_{1}, \ldots, B_{m}\right\} \subseteq \mathcal{D}(X) \backslash P$ such that $A_{1} \cap \cdots \cap A_{n} \subseteq B_{1} \cup \cdots \cup B_{m}$. As $P$ is a filter, we have $A:=A_{1} \cap \cdots \cap A_{n} \in P$. Then $\left[B_{1}\right) \cap \cdots \cap\left[B_{m}\right) \subseteq[A)$. Since $B_{1}, \ldots, B_{m} \notin P$ and $P$ is optimal, we have that $A \notin P$, which is impossible. Therefore, (5.1) is valid. Then, there exists $x \in X$ such that $x \in \bigcap\{A: A \in P\} \cap \bigcap\left\{B^{c}: B \notin P\right\}$. Now, it is straightforward to show directly that $H_{X}(x)=P$. Hence $H_{X}$ is onto.

$(2) \Rightarrow(1)$ Let $\mathcal{A}, \mathcal{B} \subseteq \mathcal{D}(X)$ be nonempty and such that $\bigcap \mathcal{A} \subseteq \cup \mathcal{B}$. We consider the filter $F$ of $\mathcal{D}(X)$ generated by $\mathcal{A}$ and the Frink ideal $I$ of $\mathcal{D}(X)$ generated by $\mathcal{B}$. Suppose that $F \cap I=\emptyset$. Since $\mathcal{D}(X)$ is an md-semilattice, it follows by Theorem 3.3 that there exists $P \in \operatorname{Opt}(\mathcal{D}(X))$ such that $F \subseteq P$ and $P \cap I=\emptyset$. Then, since the map $H_{X}$ is onto, there exists $x \in X$ such that $H_{X}(x)=P$. Thus $x \in \bigcap \mathcal{A}$; this implies that there exists $B \in \mathcal{B}$ such that $x \in B$, which is impossible. Therefore, $F \cap I \neq \emptyset$. Then, there exists $C \in F \cap I$. It follows that there are finite subsets $\left\{A_{1}, \ldots, A_{n}\right\} \subseteq \mathcal{A}$ and $\left\{B_{1}, \ldots, B_{m}\right\} \subseteq \mathcal{B}$ such that 
$A_{1} \cap \cdots \cap A_{n} \subseteq C$ and $\left[B_{1}\right) \cap \cdots \cap\left[B_{m}\right) \subseteq[C)$. By condition (4) of Definition 5.1. the last inclusion is equivalent to $C \subseteq B_{1} \cup \cdots \cup B_{m}$ and hence, we obtain that $A_{1} \cap \cdots \cap A_{n} \subseteq B_{1} \cup \cdots \cup B_{m}$.

Lemma 5.6. If $\langle X, \mathcal{T}, \mathcal{K}\rangle$ is an md-space, then $\langle X, \mathcal{T}\rangle$ is a sober space.

Proof. Let $F$ be an irreducible closed subset of $X$. Consider the set

$$
P_{F}=\{A \in \mathcal{D}(X): F \subseteq A\} .
$$

It is straightforward to show that $P_{F}$ is a filter of $\mathcal{D}(X)$. Now let $B_{1}, \ldots, B_{n} \notin P_{F}$ and let $B \in \mathcal{D}(X)$ be such that $\left[B_{1}\right) \cap \cdots \cap\left[B_{n}\right) \subseteq[B)$. Suppose that $B \in P_{F}$. That is, $F \subseteq B$. By condition (4) of Definition 5.1, we have $B \subseteq B_{1} \cup \cdots \cup B_{n}$ and this implies that $F \subseteq B_{1} \cup \cdots \cup B_{n}$. Since $F$ is irreducible, it follows that $F \subseteq B_{i}$ for some $i \in\{1, \ldots, n\}$. Then $B_{i} \in P_{F}$, which is a contradiction. Hence $B \notin P_{F}$. Thus, we have proved that $P_{F}$ is an optimal filter or $P_{F}=\mathcal{D}(X)$. That is, $P_{F} \in \mathrm{X}(\mathcal{D}(X))$.

Now by Theorem 5.5, there exists $x \in X$ such that $H_{X}(x)=P_{F}$. We show that $F=\operatorname{cl}(x)$. Given that $H_{X}(x)=P_{F}$ and $\mathcal{K}$ is a subbase of $X$, we have $x \in F$. So $\operatorname{cl}(x) \subseteq F$. Now, let $y \in F$. Let $B \in \mathcal{D}(X)$ be such that $x \in B$. Thus $B \in H_{X}(x)=P_{F}$. So, we have $F \subseteq B$ and hence $y \in B$. Then, we obtain that $y \in \operatorname{cl}(x)$. Hence, $F \subseteq \operatorname{cl}(x)$. Thus, $F=\operatorname{cl}(x)$. Therefore, since $X$ is a $T_{0}$-space, $X$ is a sober space.

Let $\langle X, \mathcal{T}, \mathcal{K}\rangle$ be an md-space. Since $\mathcal{D}(X)$ is an md-semilattice, we can consider its dual md-space

$$
\mathrm{X}(\mathcal{D}(X))=\left\langle\mathrm{X}(\mathcal{D}(X)), \mathcal{T}_{\mathcal{D}(X)}, \mathcal{K}_{\mathcal{D}(X)}\right\rangle
$$

as defined in the previous section, with

$$
\mathrm{X}(\mathcal{D}(X))=\operatorname{Opt}(\mathcal{D}(X)) \cup\{\mathcal{D}(X)\} \quad \text { and } \quad \mathcal{K}_{\mathcal{D}(X)}=\left\{\varphi_{L}(A)^{c}: A \in \mathcal{D}(X)\right\} .
$$

Moreover, for every $A \in \mathcal{D}(X), \varphi_{L}(A)=\{P \in \mathrm{X}(\mathcal{D}(X)): A \in P\}$.

Proposition 5.7. For every $m d$-space $\langle X, \mathcal{T}, \mathcal{K}\rangle$, the map $H_{X}$ from $X$ onto $\mathrm{X}(\mathcal{D}(X))$ is a homeomorphism and $\mathcal{K}_{\mathcal{D}(X)}=\left\{H_{X}[U]: U \in \mathcal{K}\right\}$.

Proof. Since $X$ is a $T_{0}$-space, it follows that $H_{X}$ is injective; by Theorem 5.5 , we obtain that $H_{X}$ is onto. A subbasic open set of the space $\mathrm{X}(\mathcal{D}(X))$ is of the form $\varphi_{L}(A)^{c}=\{P \in \mathrm{X}(\mathcal{D}(X)): A \notin P\}$ with $A \in \mathcal{D}(X)$. For every $x \in X$, we have that

$$
x \in H_{X}^{-1}\left[\varphi_{L}(A)^{c}\right] \Longleftrightarrow H_{X}(x) \in \varphi_{L}(A)^{c} \Longleftrightarrow A \notin H_{X}(x) \Longleftrightarrow x \in A^{c} .
$$

Then $H_{X}^{-1}\left[\varphi_{L}(A)^{c}\right]=A^{c} \in \mathcal{K}$ and therefore the map $H_{X}$ is continuous. In order to prove that $H_{X}$ is an open map, let $U \in \mathcal{K}$. For every $P \in \mathrm{X}(\mathcal{D}(X))$, we have

$$
\begin{aligned}
P \in H_{X}[U] \Longleftrightarrow P=H_{X}(x) \text { for some } x \in U & \\
& \Longleftrightarrow U^{c} \notin P \Longleftrightarrow P \in \varphi_{L}\left(U^{c}\right)^{c} .
\end{aligned}
$$

Then, $H_{X}[U]=\varphi_{L}\left(U^{c}\right)^{c} \in \mathcal{K}_{\mathcal{D}(X)}$ and hence $H_{X}$ is an open map. This completes the proof. 
We have shown that the corresponding dual topological structures to the mdsemilattices are the md-spaces. Now we focus on the morphisms between mdspaces that correspond to the strong homomorphisms between md-semilattices that preserve top element.

Definition 5.8. Let $\left\langle X_{1}, \mathcal{T}_{1}, \mathcal{K}_{1}\right\rangle$ and $\left\langle X_{2}, \mathcal{T}_{2}, \mathcal{K}_{2}\right\rangle$ be md-spaces. We say that a map $f: X_{1} \rightarrow X_{2}$ is strongly continuous if for every $V \in \mathcal{K}_{2}$, we have $f^{-1}[V] \in \mathcal{K}_{1}$.

Observe that each strongly continuous map is in particular a continuous map.

Proposition 5.9. Let $\left\langle X_{1}, \mathcal{T}_{1}, \mathcal{K}_{1}\right\rangle$ and $\left\langle X_{2}, \mathcal{T}_{2}, \mathcal{K}_{2}\right\rangle$ be md-spaces and let $f: X_{1} \rightarrow$ $X_{2}$ be a strongly continuous map. Then, the map $f^{-1}: \mathcal{D}_{\mathcal{K}_{2}}\left(X_{2}\right) \rightarrow \mathcal{D}_{\mathcal{K}_{1}}\left(X_{1}\right)$ is a strong homomorphism preserving the top elements.

Proof. It is clear that $f^{-1}$ is a homomorphism preserving the top elements between the meet-semilattices $\mathcal{D}_{\mathcal{K}_{2}}\left(X_{2}\right)$ and $\mathcal{D}_{\mathcal{K}_{1}}\left(X_{1}\right)$. Let $B_{1}, \ldots, B_{n}, B \in \mathcal{D}_{\mathcal{K}_{2}}\left(X_{2}\right)$ be such that $\left[B_{1}\right) \cap \cdots \cap\left[B_{n}\right) \subseteq[B)$. By condition (4) of Definition 5.1 we have $B \subseteq B_{1} \cup \cdots \cup B_{n}$. Then, $f^{-1}(B) \subseteq f^{-1}\left(B_{1}\right) \cup \cdots \cup f^{-1}\left(B_{n}\right)$. Using again condition (4) we obtain that $\left[f^{-1}\left(B_{1}\right)\right) \cap \cdots \cap\left[f^{-1}\left(B_{n}\right)\right) \subseteq\left[f^{-1}(B)\right)$. Therefore, $f^{-1}$ is a strong homomorphism.

Remark 5.10. From Proposition 5.7, it follows that for every md-space $X$, the homeomorphism $H_{X}: X \rightarrow \mathrm{X}(\mathcal{D}(X))$ and its inverse image $H_{X}^{-1}$ are strongly continuous maps.

Let $\left\langle X, \mathcal{T}_{X}, \mathcal{K}_{X}\right\rangle$ and $\left\langle Y, \mathcal{T}_{Y}, \mathcal{K}_{Y}\right\rangle$ be topological spaces with subbases. Let us say that a map $f: X \rightarrow Y$ is a strong homeomorphism if $f$ is a homeomorphism from $\left\langle X, \mathcal{T}_{X}\right\rangle$ onto $\left\langle Y, \mathcal{T}_{Y}\right\rangle$ and $\mathcal{K}_{Y}=\left\{f[U]: U \in \mathcal{K}_{X}\right\}$.

Proposition 5.11. Let $L$ and $M$ be md-semilattices and let $h: L \rightarrow M$ be a strong homomorphism preserving top elements. Then, the map $h^{-1}: \mathrm{X}(M) \rightarrow \mathrm{X}(L)$ is a strongly continuous map from $\mathrm{X}(M)$ to $\mathrm{X}(L)$.

Proof. By Proposition 3.5, we know that $h^{-1}$ is well defined. Let $a \in L$ and $Q \in \mathrm{X}(M)$. Then, we have

$$
\begin{aligned}
Q \in\left(h^{-1}\right)^{-1}\left[\varphi_{L}(a)^{c}\right] & \Longleftrightarrow h^{-1}(Q) \in \varphi_{L}(a)^{c} \\
& \Longleftrightarrow a \notin h^{-1}(Q) \\
& \Longleftrightarrow h(a) \notin Q \Longleftrightarrow Q \in \varphi_{L}(h(a))^{c} .
\end{aligned}
$$

Hence, $\left(h^{-1}\right)^{-1}\left[\varphi_{L}(a)^{c}\right]=\varphi(h(a))^{c} \in \mathcal{K}_{M}$. Therefore $h^{-1}$ is a strongly continuous map from md-space $\mathrm{X}(M)$ to $\mathrm{X}(L)$.

Let us denote by $\mathbb{T M D}$ the category whose objects are the md-semilattices with a top element and the morphisms are the strong homomorphisms preserving top elements. Let $\mathbb{M D S}$ be the category of all md-spaces and all strongly continuous maps. It is clear that in both categories TMD and $\mathbb{M D S}$ the composition is the standard composition of maps, and the identity morphisms are the identity maps. Notice that the isomorphisms of the category $\mathbb{T M D}$ are the meet-isomorphisms and the isomorphisms of the category $\mathbb{M D S}$ are the strong homeomorphisms. Now we are in condition to present the main result of this article. 

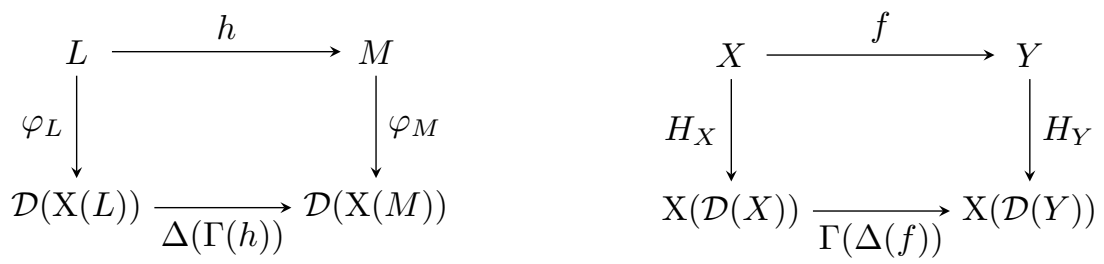

Figure 1. Commutative diagrams of morphisms in $\mathbb{T M D}$ and $\mathbb{M D}$ S.

Theorem 5.12. The categories $\mathbb{T M D}$ and $\mathbb{M D S}$ are dually equivalent via the following functors:

(1) $\Gamma: \mathbb{T M D} \rightarrow \mathbb{M D S}$ is defined by:

- For each md-semilattice $L, \Gamma(L)=\left\langle\mathrm{X}(L), \mathcal{T}_{L}, \mathcal{K}_{L}\right\rangle$;

- if $h: L \rightarrow M$ is a morphism of $\mathbb{T M D}$, then $\Gamma(h): \mathrm{X}(M) \rightarrow \mathrm{X}(L)$ is given by $\Gamma(h)=h^{-1}$;

(2) $\Delta: \mathbb{M D S} \rightarrow \mathbb{T M D}$ is defined by:

- For each md-space $\langle X, \mathcal{T}, \mathcal{K}\rangle, \Delta(X)=\left\langle\mathcal{D}_{\mathcal{K}}(X), \cap, X\right\rangle$;

- for every morphism $f: X \rightarrow Y$ of $\mathbb{M D S}, \Delta(f): \Delta(Y) \rightarrow \Delta(X)$ is defined by $\Delta(f)=f^{-1}$.

Proof. Notice that it is straightforward to check directly that $\Gamma(j \circ h)=\Gamma(h) \circ \Gamma(j)$ whenever $h: L \rightarrow M$ and $j: M \rightarrow N$ are morphisms of TMD and $\Delta(g \circ f)=\Delta(f) \circ$ $\Delta(g)$ if $f: X \rightarrow Y$ and $g: Y \rightarrow Z$ are morphisms of MDS; moreover $\Gamma\left(\operatorname{id}_{L}\right)=\operatorname{id}_{\mathrm{X}}(L)$ and $\Delta\left(\operatorname{id}_{X}\right)=\operatorname{id}_{\mathcal{D}(X)}$ for every md-semilattice $L$ and every md-space $\langle X, \mathcal{T}, \mathcal{K}\rangle$. Hence, by Propositions 5.2 and 5.3 , we obtain that $\Gamma$ and $\Delta$ are functors. Now, we only need to prove that, for every morphism $h: L \rightarrow M$ of the category $\mathbb{T M D}$ and for every morphism $f: X \rightarrow Y$ of the category $\mathbb{M D S}$ the diagrams in Figure 1 commute.

To this end, let $a \in L$. For $Q \in \mathrm{X}(M)$, we have,

$$
\begin{aligned}
& Q \in\left(\Delta(\Gamma(h)) \circ \varphi_{L}\right)(a) \Longleftrightarrow Q \in(\Gamma(h))^{-1}\left(\varphi_{L}(a)\right) \Longleftrightarrow \Gamma(h)(Q) \in \varphi_{L}(a) \\
& \Longleftrightarrow h^{-1}(Q) \in \varphi_{L}(a) \Longleftrightarrow a \in h^{-1}(Q) \Longleftrightarrow h(a) \in Q \\
& \Longleftrightarrow Q \in \varphi_{M}(h(a)) \Longleftrightarrow Q \in\left(\varphi_{M} \circ h\right)(a) \text {. }
\end{aligned}
$$

Hence, $\Delta(\Gamma(h)) \circ \varphi_{L}=\varphi_{M} \circ h$. Now, let $x \in X$. For $B \in \mathcal{D}(Y)$, we have,

$$
\begin{aligned}
B \in\left(\Gamma(\Delta(f)) \circ H_{X}\right)(x) \Longleftrightarrow B \in(\Delta(f))^{-1}\left(H_{X}(x)\right) & \Longleftrightarrow f^{-1}(B) \in H_{X}(x) \Longleftrightarrow x \in f^{-1}(B) \\
\Longleftrightarrow \Delta(f)(B) \in H_{X}(x) & \left.\Longleftrightarrow f^{\prime}\right) \\
\Longleftrightarrow f(x) \in B & \Longleftrightarrow B \in H_{Y}(f(x)) \Longleftrightarrow B \in\left(H_{Y} \circ f\right)(x) .
\end{aligned}
$$

Thus $\Gamma(\Delta(f)) \circ H_{X}=H_{Y} \circ f$. Therefore, the functors $\Gamma$ and $\Delta$ are dual equivalences and thus the categories TMD and $\mathbb{M D S}$ are dually equivalent.

We end this section with the following consequence of the previous duality theorem. We leave the details to the reader. 
Proposition 5.13. Let $L$ and $M$ be md-semilattices and $h: L \rightarrow M$ a strong homomorphism preserving top element. Then:

(1) $h$ is injective iff $h^{-1}: \mathrm{X}(M) \rightarrow \mathrm{X}(L)$ is onto;

(2) $h$ is onto iff $h^{-1}$ is injective;

(3) $h$ is a meet-isomorphism iff $h^{-1}$ is a strong homeomorphism.

\section{Subbasic Saturation AND the CONNECTiOn Between Filters AND CLOSED SETS}

In this section, we establish the connections between Frink ideals and filters of an md-semilattice and particular saturated subsets and closed subsets of its dual md-space, respectively.

Definition 6.1. Let $\langle X, \mathcal{T}, \mathcal{K}\rangle$ be an md-space. A subset $Y \subseteq X$ is called a saturated subbasic subset of $X$ if it is an intersection of subbasic open sets of $\mathcal{K}$, i.e., $Y=\bigcap\{U \in \mathcal{K}: Y \subseteq U\}$.

Let us denote by $\operatorname{Sat}_{\mathcal{K}}(X)$ the set of all saturated subbasic subsets of $\langle X, \mathcal{T}, \mathcal{K}\rangle$. It is straightforward to show directly that $\operatorname{Sat}_{\mathcal{K}}(X)$ is closed under arbitrary intersections. Then, it is a closure system. We denote by Sat $\mathcal{K}_{\mathcal{K}}($.$) the closure operator$ associated with the closure system $\operatorname{Sat}_{\mathcal{K}}(X)$. Thus, for every subset $Y \subseteq X$, $\operatorname{Sat}_{\mathcal{K}}(Y)$ is the smallest saturated subbasic subset of $X$ containing $Y$. If $Y=\{y\}$, we write $\operatorname{Sat}_{\mathcal{K}}(y)$ instead of $\operatorname{Sat}_{\mathcal{K}}(\{y\})$. Notice that $\left\langle\operatorname{Sat}_{\mathcal{K}}(X), \cap, \vee, \emptyset, X\right\rangle$, where $\bigvee_{i \in I} Y_{i}=\operatorname{Sat}_{\mathcal{K}}\left(\bigcup_{i \in I} Y_{i}\right)$, is a complete lattice.

Theorem 6.2. Let $L$ be an md-semilattice and $\left\langle\mathrm{X}(L), \mathcal{T}_{L}, \mathcal{K}_{L}\right\rangle$ its dual md-space. Then, the map $\beta: \operatorname{Fld}(L) \rightarrow \operatorname{Sat}_{\mathcal{K}_{L}}(\mathrm{X}(L))$ defined by

$$
\beta(I)=\{P \in \mathrm{X}(L): P \cap I=\emptyset\}
$$

for each $I \in \operatorname{FId}(L)$ is a dual lattice isomorphism.

Proof. It is clear that $\beta$ is well-defined because $\beta(I)=\bigcap\left\{\varphi(a)^{c}: a \in I\right\} \in$ Sat $_{\mathcal{K}_{L}}(\mathrm{X}(L))$ for every $I \in \operatorname{Fld}(L)$. Now we prove that $I \subseteq J$ iff $\beta(J) \subseteq \beta(I)$ for each $I, J \in \operatorname{Fld}(L)$. On the one hand, if $I \subseteq J$, then by definition of $\beta$ it follows that $\beta(J) \subseteq \beta(I)$. On the other hand, if we suppose that $I \nsubseteq J$, then there exists $a \in I$ such that $a \notin J$. Thus, there exists $Q \in \operatorname{Opt}(L)$ such that $a \in Q$ and $Q \cap J=\emptyset$. Then, $Q \in \beta(J)$ and $Q \notin \beta(I)$. This implies that $\beta(I) \nsubseteq \beta(J)$. Hence $\beta$ is a dual order-embedding.

Lastly, we prove that $\beta$ is onto. Let $Y \in \operatorname{Sat}_{\mathcal{K}_{L}}(\mathrm{X}(L))$. Thus, there exists $B \subseteq L$ such that $Y=\bigcap\left\{\varphi(a)^{c}: a \in B\right\}$. Consider the Frink ideal $I$ of $L$ generated by $B$, i.e.,

$$
I=\langle B\rangle=\left\{b \in L:\left(\exists\left\{b_{1}, \ldots, b_{n}\right\} \subseteq B\right)\left(\left[b_{1}\right) \cap \cdots \cap\left[b_{n}\right) \subseteq[b)\right)\right\} .
$$

We prove that $\beta(I)=Y$. Let $P \in \beta(I)$. Then $I \cap P=\emptyset$ and thus $B \cap P=\emptyset$. We thus obtain $P \in \bigcap\left\{\varphi(b)^{c}: a \in B\right\}=Y$. So, $\beta(I) \subseteq Y$. In order to prove the inverse inclusion, let $P \in Y$. Suppose that $P \notin \beta(I)$, i.e., $I \cap P \neq \emptyset$. Then, there exists $a \in P$ and $b_{1}, \ldots, b_{n} \in B$ such that $\left[b_{1}\right) \cap \cdots \cap\left[b_{n}\right) \subseteq[a)$. As $P$ is optimal, we have that $b_{i} \in P$ for some $b_{i} \in\left\{b_{1}, \ldots, b_{n}\right\}$. Then, $P \in \varphi\left(b_{i}\right)$ and thus 
$P \notin \bigcap\left\{\varphi(b)^{c}: b \in B\right\}=Y$, which is a contradiction. So, $P \in \beta(I)$ and hence we have $Y \subseteq \beta(I)$. Therefore $Y=\beta(I)$. This completes the proof.

Let $\langle X, \mathcal{T}, \mathcal{K}\rangle$ be an md-space. Recall that $\mathcal{D}_{\mathcal{K}}(X)=\left\{U^{c}: U \in \mathcal{K}\right\}$. We denote by $\mathcal{C}_{\mathcal{K}}(X)$ the family of all closed subsets of $X$ that are arbitrary intersections of elements of $\mathcal{D}_{\mathcal{K}}(X)$. That is, $\mathcal{C}_{\mathcal{K}}(X)$ is the closure system on $X$ generated by the family $\mathcal{D}_{\mathcal{K}}(X)$. So, $\mathcal{C}_{\mathcal{K}}(X)$ is a complete lattice. The elements of $\mathcal{C}_{\mathcal{K}}(X)$ are called subbasic closed subsets.

Let $L$ be an md-semilattice and $\mathrm{X}(L)$ its dual md-space. It should be noted that the subbasic closed subsets of $\mathrm{X}(L)$ are of the form $\bigcap_{a \in A} \varphi(a)$ with $A \subseteq L$. We define the following maps. Let $\Phi: \mathcal{C}_{\mathcal{K}_{L}}(\mathrm{X}(L)) \rightarrow \mathrm{Fi}(L)$ be defined by

$$
\Phi(Y)=F_{Y}:=\{a \in L: Y \subseteq \varphi(a)\}
$$

for every subbasic closed $Y$ of $\mathrm{X}(L)$ and, let $\Psi: \operatorname{Fi}(L) \rightarrow \mathcal{C}_{\mathcal{K}_{L}}(\mathrm{X}(L))$ be defined by

$$
\Psi(F):=\bigcap_{a \in F} \varphi(a)=\{P \in \mathrm{X}(L): F \subseteq P\}
$$

for every filter $F$ of $L$.

Lemma 6.3. For every $Y \in \mathcal{C}_{\mathcal{K}_{L}}(\mathrm{X}(L))$ and every $F \in \mathrm{Fi}(L)$ we have that

$$
(\Psi \circ \Phi)(Y)=Y \quad \text { and } \quad(\Phi \circ \Psi)(F)=F .
$$

Proof. The first equality is clear from the definition of subbasic closed subsets. For the second one, let $F \in \operatorname{Fi}(L)$. We need to check that $F=F_{\Psi(F)}$. So, let $a \in F$ and $P \in \Psi(F)=\bigcap_{x \in F} \varphi(x)$. Then, $P \in \varphi(a)$. This implies that $\Psi(F) \subseteq \varphi(a)$ and hence $a \in F_{\Psi(F)}$. Reciprocally, let $a \in F_{\Psi(F)}$. So, $\Psi(F) \subseteq \varphi(a)$. We suppose that $a \notin F$. Then, there exists $P \in \operatorname{Opt}(L)$ such that $F \subseteq P$ and $a \notin P$. Thus, $P \in \Psi(F)$. Hence, $a \in P$. This is a contradiction. Then, $a \in F$. This completes the proof.

From the definitions of the maps $\Phi$ and $\Psi$ and by the previous lemma, we have the following result.

Theorem 6.4. The maps $\Phi$ and $\Psi$ are mutually inverse dual lattice isomorphisms.

We denote by $\mathcal{C}_{\text {irr }}(X)$ the collection of all irreducible closed subsets of a topological space $\langle X, \mathcal{T}\rangle$.

Lemma 6.5. If $\langle X, \mathcal{T}, \mathcal{K}\rangle$ is an md-space, then $\mathcal{C}_{\text {irr }}(X) \subseteq \mathcal{C}_{\mathcal{K}}(X)$.

Proof. By Theorem 5.12, we can assume that $\langle X, \mathcal{T}, \mathcal{K}\rangle$ is the dual md-space of an md-semilattice $L$ and thus, $X=\mathrm{X}(L)$ and $\mathcal{K}=\mathcal{K}_{L}=\left\{\varphi(a)^{c}: a \in L\right\}$.

Let $A \in \mathcal{C}_{\text {irr }}(X)$. Let us show that $A=\Psi\left(F_{A}\right)$, where $F_{A}=\{a \in L$ : $A \subseteq \varphi(a)\} \in \mathrm{Fi}(L)$. Let $P \in A$ and $a \in F_{A}$. Thus, $A \subseteq \varphi(a)$ and then $P \in \varphi(a)$. Hence, $P \in \Psi\left(F_{A}\right)$. Now, assume that $P \in \Psi\left(F_{A}\right)$. So, $P \in \varphi(a)$ for all $a \in F_{A}$. Since $A$ is a closed subset and $\mathcal{K}_{L}$ is a subbase, it follows that $A=\bigcap_{i \in I}\left(\varphi\left(a_{i 1}\right) \cup \cdots \cup \varphi\left(a_{i n_{i}}\right)\right)$. Because $A$ is irreducible, we obtain that for every $i \in I, A \subseteq \varphi\left(a_{i j_{i}}\right)$ for some $j_{i} \in\left\{1, \ldots, n_{i}\right\}$. So, $a_{i j_{i}} \in F_{A}$ for all $i \in I$. Then, $P \in \varphi\left(a_{i j_{i}}\right)$ for all $i \in I$. Hence, we obtain that $P \in \bigcap_{i \in I}\left(\varphi\left(a_{i 1}\right) \cup \cdots \cup \varphi\left(a_{i n_{i}}\right)\right)=$ $A$. Therefore, $A=\Psi\left(F_{A}\right) \in \mathcal{C}_{\mathcal{K}}(X)$. 
We have seen that the lattice of filters of an md-semilattice $L$ is in $1-1$ correspondence with the lattice of the subbasic closed subsets of its dual md-space $\mathrm{X}(L)$. Now we want to determine the class of subbasic closed subsets that correspond to the optimal filters. To this end, we introduce a variant of irreducibility for closed subsets.

Definition 6.6. Let $\langle X, \mathcal{T}, \mathcal{K}\rangle$ be a topological space. We say that a nonempty closed set $A$ of $X$ is weakly-irreducible when for all $U, U_{1}, \ldots, U_{n} \in \mathcal{K}$,

$$
A \subseteq U^{c} \subseteq U_{1}^{c} \cup \cdots \cup U_{n}^{c} \text { implies } A \subseteq U_{i}^{c} \text { for some } i \in\{1, \ldots, n\} .
$$

For an md-space $\langle X, \mathcal{T}, \mathcal{K}\rangle$, let us denote by $\mathcal{C}_{\mathcal{K}}^{\text {wirr }}(X)$ the collection of all subbasic closed subsets that are weakly-irreducible.

Lemma 6.7. Let $\langle X, \mathcal{T}, \mathcal{K}\rangle$ be a topological space and let $A$ be a closed subset of $X$. If $A$ is irreducible, then $A$ is weakly-irreducible.

Corollary 6.8. Let $\langle X, \mathcal{T}, \mathcal{K}\rangle$ be an md-space. Then $\mathcal{C}_{\text {irr }}(X) \subseteq \mathcal{C}_{\mathcal{K}}^{\text {wirr }}(X)$.

Proof. It is an immediate consequence from the previous lemma and by Lemma 6.5

Proposition 6.9. Let $L$ be an md-semilattice and $\left\langle\mathrm{X}(L), \mathcal{T}_{L}, \mathcal{K}_{L}\right\rangle$ its dual mdspace. Then, the dual isomorphisms $\Phi$ and $\Psi$ restricted to the ordered sets $\left\langle\mathcal{C}_{\mathcal{K}_{L}}^{\text {wirr }}(\mathrm{X}(L)), \subseteq\right\rangle$ and $\langle\mathrm{X}(L), \subseteq\rangle$, respectively, are dual order-isomorphisms.

Proof. Notice that we only need to prove that $\Phi$ and $\Psi$ are well defined functions on $\mathcal{C}_{\mathcal{K}_{L}}^{\text {wirr }}(\mathrm{X}(L))$ and $\mathrm{X}(L)$, respectively. Let $Y$ be a weakly-irreducible subbasic closed subset of $\left\langle\mathrm{X}(L), \mathcal{T}_{\mathcal{K}_{L}}\right\rangle$. We need to show that the filter $\Phi(Y)=F_{Y} \in$ $\mathrm{X}(L)=\operatorname{Opt}(L) \cup\{L\}$. Let $a, a_{1}, \ldots, a_{n} \in L$ be such that $a_{1}, \ldots, a_{n} \notin F_{Y}$ and $\left[a_{1}\right) \cap \cdots \cap\left[a_{n}\right) \subseteq[a)$. So, by Remark 4.3 and Proposition 4.4 we have $\varphi(a) \subseteq$ $\varphi\left(a_{1}\right) \cup \cdots \cup \varphi\left(a_{n}\right)$. We suppose towards a contradiction that $a \in F_{Y}$. Thus $Y \subseteq \varphi(a)$. That is, $Y \subseteq \varphi(a) \subseteq \varphi\left(a_{1}\right) \cup \cdots \cup \varphi\left(a_{n}\right)$. Since $Y$ is weakly-irreducible, it follows that $Y \subseteq \varphi\left(a_{i}\right)$ for some $i \in\{1, \ldots, n\}$. Then, $a_{i} \in F_{Y}$ and this is a contradiction. Hence $a \notin F_{Y}$. Therefore, $F_{Y} \in \operatorname{Opt}(L)$ or $F_{Y}=L$, that is, $F_{Y} \in \mathrm{X}(L)$.

Now, let $P \in \mathrm{X}(L)$. We show that the subbasic closed subset $\Psi(P)$ is weaklyirreducible. If $P=L$, then $\Psi(L)=\{L\}$ and it is clearly weakly-irreducible. Assume that $P \in \operatorname{Opt}(L)$. Let $a, a_{1}, \ldots, a_{n} \in L$ be such that

$$
\Psi(P) \subseteq \varphi(a) \subseteq \varphi\left(a_{1}\right) \cup \cdots \cup \varphi\left(a_{n}\right) .
$$

Since $\varphi(a) \subseteq \varphi\left(a_{1}\right) \cup \cdots \cup \varphi\left(a_{n}\right)$, it follows that $\left[a_{1}\right) \cap \cdots \cap\left[a_{n}\right) \subseteq[a)$; moreover notice that $P \in \Psi(P)$. So, $a \in P$. Because $P$ is optimal, there exists $i=1, \ldots, n$ such that $a_{i} \in P$. Then $\Psi(P) \subseteq \varphi\left(a_{i}\right)$. Therefore, $\Psi(P)$ is a weakly-irreducible.

It is clear that the partially ordered sets $\mathcal{C}_{\mathcal{K}_{L}}^{\text {wirr }}(\mathrm{X}(L)) \backslash\{\{L\}\}$ and $\operatorname{Opt}(L)$ are dual order-isomorphic. We summarize in Table 1 the correspondence between filters of an md-semilattice $L$ and the closed subsets of its dual md-space $\langle X, \mathcal{T}, \mathcal{K}\rangle$. 


\begin{tabular}{|cccc|}
\hline md-semilattices & \multicolumn{3}{c|}{ md-spaces } \\
\hline $\mathrm{Fi}(L)$ & $\longleftrightarrow$ & $\mathcal{C}_{\mathcal{K}}(X)$ & subbasic closed subsets \\
$\cup$ & & $\cup$ & \\
$\mathrm{X}(L)$ & $\longleftrightarrow$ & $\mathcal{C}_{\mathcal{K}}^{\text {wirr }}(X)$ & weakly-irreducible subbasic closed \\
$\cup$ & & $\cup$ & \\
Opt $(L)$ & & $\mathcal{C}_{\mathcal{K}}^{\text {wirr }}(X) \backslash\{\{L\}\}$ & \\
\hline
\end{tabular}

TABLE 1. Correspondence between filters and closed subsets.

Definition 6.10. A topological space $\langle X, \mathcal{T}, \mathcal{K}\rangle$ with a subbase $\mathcal{K}$ is called $s$-sober if for every weakly-irreducible subbasic closed $A$ of $X$, there exists a unique $x \in X$ such that $\operatorname{cl}(x)=A$.

Proposition 6.11. Let $\langle X, \mathcal{T}, \mathcal{K}\rangle$ be an md-space. Then, it is s-sober.

Proof. Let $Y$ be a weakly-irreducible subbasic closed subset of $X$. Then, by Proposition 6.9. we have $F_{Y}:=\left\{A \in \mathcal{D}_{\mathcal{K}}(X): Y \subseteq A\right\} \in \mathrm{X}\left(\mathcal{D}_{\mathcal{K}}(X)\right)$. Then, by Theorem 5.5. there exists $x \in X$ such that $F_{Y}=H_{X}(x)$. Recall that $H_{X}(x)=\{A \in$ $\left.\mathcal{D}_{\mathcal{K}}(X): x \in A\right\}$. Now we want to prove that $Y=\operatorname{cl}(x)$. By Proposition 6.9 again, we have $Y=\bigcap F_{Y}=\bigcap H_{X}(x)$. Thus $x \in \bigcap H_{X}(x)=Y$. Since $Y$ is a closed subset, it follows that $\operatorname{cl}(x) \subseteq Y$. Now, let $a \in Y$ and let $B \in \mathcal{D}_{\mathcal{K}}(X)$ be such that $x \in B$. Then, $B \in H_{X}(x)=F_{Y}$; this implies that $Y \subseteq B$. Thus, $a \in B$. Since $\mathcal{K}$ is a subbase of $X$, it follows that $a \in \operatorname{cl}(x)$. Hence, $Y \subseteq \operatorname{cl}(x)$. Therefore, we have proved that $Y=\operatorname{cl}(x)$. Moreover, this $x$ is unique because the md-spaces are $T_{0}$. This completes the proof.

Proposition 6.12. Let $\langle X, \mathcal{T}, \mathcal{K}\rangle$ be a topological space with a subbase $\mathcal{K}$ that satisfies conditions (1)-(4) of Definition 5.1. If $\langle X, \mathcal{T}, \mathcal{K}\rangle$ is s-sober, then $\langle X, \mathcal{T}, \mathcal{K}\rangle$ is an md-space.

Proof. We only need to show that condition (5) of Definition 5.1 holds. To this end, we use the characterization given in Theorem 5.5. Let $F \in \mathrm{X}\left(\mathcal{D}_{\mathcal{K}}(X)\right)$. Then, by Proposition 6.9, we have that the set $A:=\bigcap F$ is a weakly-irreducible subbasic closed subset of $X$. By hypothesis, there exists $x \in X$ such that $\operatorname{cl}(x)=A$. Now, we want to prove that $H_{X}(x)=F$. Let $B \in H_{X}(x)$. So, $\operatorname{cl}(x) \subseteq B$ and then $A \subseteq B$. Thus $B^{c} \subseteq A^{c}=\bigcup\left\{C^{c}: C \in F\right\}$. Since $B^{c} \in \mathcal{K}$ is compact, we have that there are $C_{1}, \ldots, C_{n} \in F$ such that $B^{c} \subseteq C_{1}^{c} \cup \cdots \cup C_{n}^{c}$. It follows that $C_{1} \cap \cdots \cap C_{n} \subseteq B$ and since $F$ is a filter, we obtain that $B \in F$. Reciprocally, let $B \in F$. So, $A \subseteq B$ and then $\operatorname{cl}(x) \subseteq B$. Thus, $x \in B$ and hence $B \in H_{X}(x)$. We have proved that $F=H_{X}(x)$ and hence $H_{X}$ is onto. Therefore, $\langle X, \mathcal{T}, \mathcal{K}\rangle$ is an md-space.

Hence, we have characterized the md-spaces as follows:

Corollary 6.13. A topological space $\langle X, \mathcal{T}, \mathcal{K}\rangle$ with a subbase $\mathcal{K}$ is an md-space if and only if it satisfies conditions (1)-(4) of Definition 5.1 and is s-sober. 


\section{TOPOLOGICAL REPRESENTATION OF CONGRUENCES}

The purpose of this section is to represent topologically some congruences on md-semilattices. Certain congruence relations on md-semilattices were studied and described by Hickman in [12. Here we focus on a subclass of those congruences considered by Hickman.

Definition $7.1([12])$. Let $\langle L, \wedge\rangle$ be a semilattice. A semilattice congruence $\theta$ on $L$ is said to be join partial if $a_{i} \theta b_{i}$ for $i=1, \ldots, n$ and both $a_{1} \vee \cdots \vee a_{n}$ and $b_{1} \vee \cdots \vee b_{n}$ exist in $L$ then $\left(a_{1} \vee \cdots \vee a_{n}\right) \theta\left(b_{1} \vee \cdots \vee b_{n}\right)$.

For a map $f: A \rightarrow B$, we denote the kernel of $f$ by $\operatorname{Ker}(f):=\left\{\left(a_{1}, a_{2}\right) \in A^{2}\right.$ : $\left.f\left(a_{1}\right)=f\left(a_{2}\right)\right\}$. Recall that all md-semilattices have a top element.

Proposition 7.2 ([12, Proposition 4.4]). Let $L$ be an $m d$-semilattice and $\theta$ a join partial congruence on $L$. Then the canonical projection $\pi_{\theta}: L \rightarrow L / \theta$ defined by $\pi_{\theta}(a)=\bar{a}$, the congruence class of a under $\theta$, is a strong homomorphism preserving top and $\operatorname{Ker}\left(\pi_{\theta}\right)=\theta$.

Let us consider the following join partial congruences on md-semilattices that seem to be more natural as the next proposition shows.

Definition 7.3. Let $L$ be an md-semilattice. A join partial congruence $\theta$ on $L$ is called an $m d$-congruence if the quotient semilattice $L / \theta$ is mildly distributive.

In Figure 2, we depict an md-semilattice $L$ and a join partial congruence $\theta$. We can easily observe that the quotient semilattice $L / \theta$ under $\theta$ is a non-distributive lattice; in particular, $L / \theta$ is not mildly distributive and hence $\theta$ is not an mdcongruence.

Proposition 7.4. Let $L$ and $M$ be md-semilattices and let $h: L \rightarrow M$ be an onto strong homomorphism. Then, $\operatorname{Ker}(h)$ is an md-congruence on $L$ and $L / \operatorname{Ker}(h) \cong$ $M$.

Proof. It is straightforward to show directly that $\operatorname{Ker}(h)$ is a join partial congruence on $L$ (see [12, p. 297]). Let us now prove that the quotient semilattice $L / \operatorname{Ker}(h)$ is mildly distributive. Let $a_{1}, \ldots, a_{n}, a \in L$ be such that $\left[\overline{a_{1}}\right) \cap \cdots \cap\left[\overline{a_{n}}\right) \subseteq[\bar{a})$, where $\bar{x}$ denotes the congruence class of $x$ under $\operatorname{Ker}(h)$. We show that $\left[h\left(a_{1}\right)\right) \cap \cdots \cap$ $\left[h\left(a_{n}\right)\right) \subseteq[h(a))$ in $M$. Let $m \in M$ be such that $h\left(a_{i}\right) \leq m$ for all $i=1, \ldots, n$. Since $h$ is onto, it follows that $m=h(b)$ for some $b \in L$. Then $h\left(a_{i}\right)=h\left(a_{i} \wedge b\right)$ for all $i=1, \ldots, n$. So $\overline{a_{i}}=\overline{a_{i}} \wedge \bar{b}$ for all $i=1, \ldots, n$. That is, $\overline{a_{i}} \leq \bar{b}$ for all $i=1, \ldots, n$ and thus $\bar{a} \leq \bar{b}$. Then $h(a) \leq h(b)$. Now, since $M$ is mildly distributive, it follows that $h(a)=\left(h\left(a_{1}\right) \wedge h(a)\right) \vee \cdots \vee\left(h\left(a_{n}\right) \wedge h(a)\right)$. This implies that $\bar{a}=\left(\overline{a_{1}} \wedge \bar{a}\right) \vee \cdots \vee\left(\overline{a_{n}} \wedge \bar{a}\right)$. Hence $L / \operatorname{Ker}(h)$ is mildly distributive and therefore $\operatorname{Ker}(h)$ is an md-congruence.

If we define $\psi: L / \operatorname{Ker}(h) \rightarrow M$ as $\psi(\bar{a})=h(a)$, then a standard argument shows that $\psi$ is an isomorphism of semilattices.

Now we are going to characterize topologically the class of md-congruences on an md-semilattice. 


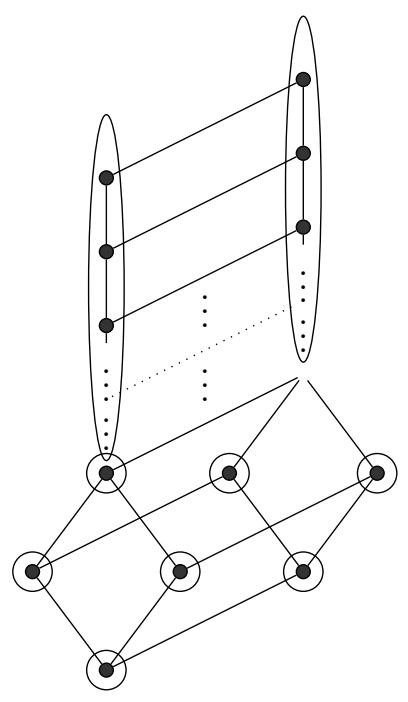

$L$

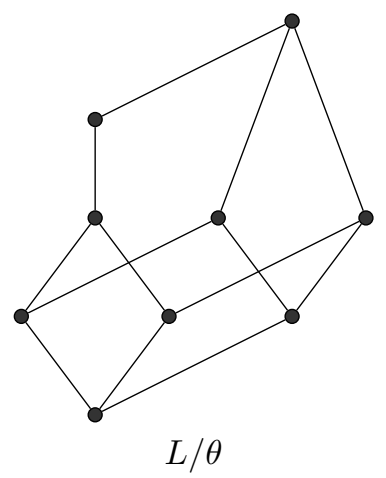

Figure 2. A partial join congruence on an md-semilattice that is not an md-congruence

Let $L$ be an md-semilattice and $\left\langle\mathrm{X}(L), \mathcal{T}_{L}, \mathcal{K}_{L}\right\rangle$ its dual md-space. Recall that $\mathrm{X}(L)=\operatorname{Opt}(L) \cup\{L\}$ and $\mathcal{K}_{L}=\left\{\varphi(a)^{c}: a \in L\right\}$. Let $\theta$ be an md-congruence on $L$. Since $L / \theta$ is an md-semilattice, we can consider its dual md-space

$$
\left\langle\mathrm{X}(L / \theta), \mathcal{T}_{L / \theta}, \mathcal{K}_{L / \theta}\right\rangle
$$

where

$$
\mathrm{X}(L / \theta)=\operatorname{Opt}(L / \theta) \cup\{L / \theta\} \quad \text { and } \quad \mathcal{K}_{L / \theta}=\left\{\varphi(a / \theta)^{c}: a / \theta \in L / \theta\right\} .
$$

We already know that the canonical projection $\pi_{\theta}: L \rightarrow L / \theta$ is an onto strong homomorphism preserving the top element. Then, by Proposition 5.13 we obtain that $\pi_{\theta}^{-1}: \mathrm{X}(L / \theta) \rightarrow \mathrm{X}(L)$ is an injective strongly continuous map. Let

$$
\left.X_{\theta}:=\pi_{\theta}^{-1}[X(L / \theta)]=\left\{\pi_{\theta}^{-1}(Q): Q \in \mathrm{X}(L / \theta)\right\} \subseteq \mathrm{X}(L)\right)^{\dagger}
$$

Let $\left\langle X_{\theta}, \mathcal{T}_{\theta}\right\rangle$ be the subspace of $\left\langle\mathrm{X}(L), \mathcal{T}_{L}\right\rangle$, that is, $\mathcal{T}_{\theta}=\left\{U \cap X_{\theta}: U \in \mathcal{T}_{L}\right\}$ is the induced topology by $X_{\theta}$. Let $\mathcal{K}_{\theta}:=\left\{\varphi(a)^{c} \cap X_{\theta}: \varphi(a)^{c} \in \mathcal{K}_{L}\right\}=\left\{\varphi(a)^{c} \cap X_{\theta}\right.$ : $a \in L\}$. Since $\mathcal{K}_{L}$ is a subbase for the space $\mathrm{X}(L)$, it follows that $\mathcal{K}_{\theta}$ is a subbase for the subspace $\left\langle X_{\theta}, \mathcal{T}_{\theta}\right\rangle$. We want to show that $\left\langle X_{\theta}, \mathcal{T}_{\theta}, \mathcal{K}_{\theta}\right\rangle$ is an md-space. We need the following result that is easy to check, and thus we leave the details to the reader.

Lemma 7.5. Let $\langle X, \mathcal{T}, \mathcal{K}\rangle$ be an md-space and let $\left\langle Y, \mathcal{T}^{\prime}, \mathcal{K}^{\prime}\right\rangle$ be an arbitrary topological space with a subbase $\mathcal{K}^{\prime}$. If there is a strong homeomorphism $f: X \rightarrow Y$, then $\left\langle Y, \mathcal{T}^{\prime}, \mathcal{K}^{\prime}\right\rangle$ is an md-space.

\footnotetext{
${ }^{\dagger}$ We refer the reader to Section 2 for the set theoretical conventions.
} 
Proposition 7.6. The map $\pi_{\theta}^{-1}: \mathrm{X}(L / \theta) \rightarrow X_{\theta}$ is a strong homeomorphism from the md-space $\left\langle\mathrm{X}(L / \theta), \mathcal{T}_{L / \theta}, \mathcal{K}_{L / \theta}\right\rangle$ onto the topological structure $\left\langle X_{\theta}, \mathcal{T}_{\theta}, \mathcal{K}_{\theta}\right\rangle$. Therefore, $\left\langle X_{\theta}, \mathcal{T}_{\theta}, \mathcal{K}_{\theta}\right\rangle$ is an md-space.

Proof. It is clear that $\pi_{\theta}^{-1}: \mathrm{X}(L / \theta) \rightarrow X_{\theta}$ is a bijective strongly continuous map. Let us now to prove that $\mathcal{K}_{\theta}=\left\{\pi_{\theta}^{-1}\left[\varphi(a / \theta)^{c}\right]: a \in L\right\}$.

Let $a \in L$ and $\pi_{\theta}^{-1}(Q) \in X_{\theta}$. Then

$$
\begin{aligned}
& \pi_{\theta}^{-1}(Q) \in \pi_{\theta}^{-1}\left[\varphi(a / \theta)^{c}\right] \Longleftrightarrow\left(\exists Q^{\prime} \in \varphi(a / \theta)^{c}\right)\left(\pi_{\theta}^{-1}\left(Q^{\prime}\right)=\pi_{\theta}^{-1}(Q)\right) \\
& \Longleftrightarrow Q \in \varphi(a / \theta)^{c} \Longleftrightarrow \pi_{\theta}^{-1}(Q) \in \varphi(a)^{c} \cap X_{\theta} .
\end{aligned}
$$

Thus $\pi_{\theta}^{-1}\left[\varphi(a / \theta)^{c}\right]=\varphi(a)^{c} \cap X_{\theta}$. Hence, $\mathcal{K}_{\theta}=\left\{\pi_{\theta}^{-1}\left[\varphi(a / \theta)^{c}\right]: a \in L\right\}=\left\{\pi_{\theta}^{-1}[U]\right.$ : $\left.U \in \mathcal{K}_{L / \theta}\right\}$. Then $\pi_{\theta}^{-1}: \mathrm{X}(L / \theta) \rightarrow X_{\theta}$ is a strong homeomorphism. Therefore, by the previous lemma, $\left\langle X_{\theta}, \mathcal{T}_{\theta}, \mathcal{K}_{\theta}\right\rangle$ is an md-space.

Definition 7.7. Let $\langle X, \mathcal{T}, \mathcal{K}\rangle$ be an md-space and let $\left\langle Y, \mathcal{T}^{\prime}, \mathcal{K}^{\prime}\right\rangle$ be a topological space with a subbase $\mathcal{K}^{\prime}$. We shall say that $\left\langle Y, \mathcal{T}^{\prime}, \mathcal{K}^{\prime}\right\rangle$ is an md-subspace of $\langle X, \mathcal{T}, \mathcal{K}\rangle$ if $\left\langle Y, \mathcal{T}^{\prime}\right\rangle$ is a topological subspace of $\langle X, \mathcal{T}\rangle$ and $\mathcal{K}^{\prime}=\{U \cap Y: U \in \mathcal{K}\}$.

By the previous proposition, we obtain the following result.

Corollary 7.8. Let $L$ be an md-semilattice and $\theta$ an $m d$-congruence on $L$. Then $\left\langle X_{\theta}, \mathcal{T}_{\theta}, \mathcal{K}_{\theta}\right\rangle$ is an md-subspace of $\left\langle\mathrm{X}(L), \mathcal{T}_{L}, \mathcal{K}_{L}\right\rangle$.

Now let $\left\langle Y, \mathcal{T}^{\prime}, \mathcal{K}^{\prime}\right\rangle$ be an md-subspace of an md-space $\langle X, \mathcal{T}, \mathcal{K}\rangle$. Consider the corresponding dual md-semilattices $\left\langle\mathcal{D}_{\mathcal{K}}(X), \cap, X\right\rangle$ and $\left\langle\mathcal{D}_{\mathcal{K}^{\prime}}(Y), \cap, Y\right\rangle$. Since $\mathcal{K}^{\prime}=\{U \cap Y: U \in \mathcal{K}\}$ and $\mathcal{D}_{\mathcal{K}^{\prime}}(Y)=\{Y \backslash(U \cap Y): U \in \mathcal{K}\}$, it follows that $\mathcal{D}_{\mathcal{K}^{\prime}}(Y)=\left\{A \cap Y: A \in \mathcal{D}_{\mathcal{K}}(X)\right\}$. For notational convenience, let us denote the elements of the md-semilattice $\mathcal{D}_{\mathcal{K}}(X)$ with lower case letters $a, b, c$, etc. Let us define the binary relation $\theta_{Y}$ on $\mathcal{D}_{\mathcal{K}}(X)$ as follows: For every $a, b \in \mathcal{D}_{\mathcal{K}}(X)$,

$$
a \theta_{Y} b \text { if and only if } a \cap Y=b \cap Y .
$$

It is obvious that $\theta_{Y}$ is an equivalence relation on $\mathcal{D}_{\mathcal{K}}(X)$.

Proposition 7.9. The relation $\theta_{Y}$ is an md-congruence on the md-semilattice $\mathcal{D}_{\mathcal{K}}(X)$.

Proof. It is clear that $\theta_{Y}$ is a congruence with respect to the meet $\cap$. Moreover, since the supremum in $\mathcal{D}_{\mathcal{K}}(X)$, when it exists, is the union, it follows that $\theta_{Y}$ is in fact a join partial congruence. It should be noted that for every $a, b \in \mathcal{D}_{\mathcal{K}}(X)$, $a / \theta_{Y} \leq b / \theta_{Y} \Longleftrightarrow a \cap Y \subseteq b \cap Y$.

Now we prove that the quotient semilattice $\mathcal{D}_{\mathcal{K}}(X) / \theta_{Y}$ is mildly distributive. For this, let $a_{1}, \ldots, a_{n}, a \in \mathcal{D}_{\mathcal{K}}(X)$ be such that $\left[a_{1} / \theta_{Y}\right) \cap \cdots \cap\left[a_{n} / \theta_{Y}\right) \subseteq\left[a / \theta_{Y}\right)$. We need to prove that $a / \theta_{Y}=\left(a_{1} / \theta_{Y} \wedge a / \theta_{Y}\right) \vee \cdots \vee\left(a_{n} / \theta_{Y} \wedge a / \theta_{Y}\right)$. First, we show that $\left[a_{1} \cap Y\right) \cap \cdots \cap\left[a_{n} \cap Y\right) \subseteq[a \cap Y)$ in $\mathcal{D}_{\mathcal{K}^{\prime}}(Y)$. Let $b \cap Y \in \mathcal{D}_{\mathcal{K}^{\prime}}(Y)$ be such that $a_{i} \cap Y \subseteq b \cap Y$ for all $i=1, \ldots, n$. Thus $a_{i} / \theta_{Y} \leq b / \theta_{Y}$ for all $i=1, \ldots, n$ and then $a / \theta_{Y} \leq b / \theta_{Y}$. Hence, since $\mathcal{D}_{\mathcal{K}^{\prime}}(Y)$ is mildly distributive, it follows that

$$
\begin{aligned}
a \cap Y & =\left[\left(a_{1} \cap Y\right) \cap(a \cap Y)\right] \cup \cdots \cup\left[\left(a_{n} \cap Y\right) \cap(a \cap Y)\right] \\
& =\left(a_{1} \cap a \cap Y\right) \cup \cdots \cup\left(a_{n} \cap a \cap Y\right) .
\end{aligned}
$$


It is clear that $a / \theta_{Y}$ is an upper bound of $a_{i} / \theta_{Y} \wedge a / \theta_{Y}$ for $i=1, \ldots, n$. Let $b \in$ $\mathcal{D}_{\mathcal{K}}(X)$ be such that $a_{i} / \theta_{Y} \wedge a / \theta_{Y} \leq b / \theta_{Y}$ for all $i=1, \ldots, n$. Then $a_{i} \cap a \cap Y \subseteq b \cap Y$ for all $i=1, \ldots, n$. From (7.1), it follows that $a \cap Y \subseteq b \cap Y$ and thus $a / \theta_{Y} \leq b / \theta_{Y}$. So, we have proved that $a / \theta_{Y}=\left(a_{1} / \theta_{Y} \wedge a / \theta_{Y}\right) \vee \cdots \vee\left(a_{n} / \theta_{Y} \wedge a / \theta_{Y}\right)$. Hence $\mathcal{D}_{\mathcal{K}}(X) / \theta_{Y}$ is an md-semilattice and therefore $\theta_{Y}$ is an md-congruence.

Let $L$ be an md-semilattice and $\langle X, \mathcal{T}, \mathcal{K}\rangle$ its dual md-space. We have defined the correspondences between md-congruences on $L$ and md-subspaces of $X$ by:

$$
\theta \mapsto\left\langle X_{\theta}, \mathcal{T}_{\theta}, \mathcal{K}_{\theta}\right\rangle \quad \text { and } \quad\left\langle Y, \mathcal{T}^{\prime}, \mathcal{K}^{\prime}\right\rangle \mapsto \theta_{Y}
$$

Lastly, we want to show that these correspondences are mutually inverse in the following sense: Let us prove that for every md-congruence $\theta$ on $L$,

$$
a \theta b \Longleftrightarrow \varphi_{L}(a) \theta_{X_{\theta}} \varphi_{L}(b)
$$

and for every md-subspace $\left\langle Y, \mathcal{T}^{\prime}, \mathcal{K}^{\prime}\right\rangle$ of $\langle X, \mathcal{T}, \mathcal{K}\rangle$,

$$
Y \text { and } X_{\theta_{Y}} \text { are strong homeomorphic. }
$$

Assume that $a \theta b$ and let $\pi_{\theta}^{-1}(Q) \in X_{\theta}$. Then

$$
\pi_{\theta}^{-1}(Q) \in \varphi_{L}(a) \Longleftrightarrow a / \theta \in Q \Longleftrightarrow b / \theta \in Q \Longleftrightarrow \pi_{\theta}^{-1}(Q) \in \varphi_{L}(b)
$$

Thus $\varphi_{L}(a) \cap X_{\theta}=\varphi_{L}(b) \cap X_{\theta}$ and hence $\varphi_{L}(a) \theta_{X_{\theta}} \varphi_{L}(b)$. Now suppose that $a / \theta \neq b / \theta$. We can assume that $a / \theta \not b b / \theta$. Since $L / \theta$ is an md-semilattice, there exists $Q \in \mathrm{X}(L / \theta)$ such that $a / \theta \in Q$ and $b / \theta \notin Q$. Thus $a \in \pi_{\theta}^{-1}(Q)$ and $b \notin \pi_{\theta}^{-1}(Q)$. Then $\varphi_{L}(a) \cap X_{\theta} \nsubseteq \varphi_{L}(b) \cap X_{\theta}$ and hence $\varphi_{L}(a) / \theta_{X_{\theta}} \neq \varphi_{L}(b) / \theta_{X_{\theta}}$. Therefore 7.2 holds.

In order to prove 7.3 , we consider the map $e: L \rightarrow \mathcal{D}_{\mathcal{K}^{\prime}}(Y)$ defined by $e(a)=$ $\varphi_{L}(a) \cap Y$. It is straightforward to show that $e$ is an onto strong homomorphism and $\operatorname{Ker}(e)=\theta_{Y}$. Then, by Proposition 7.4 we have $L / \theta_{Y}$ and $\mathcal{D}_{\mathcal{K}^{\prime}}(Y)$ are isomorphic. Thus, by Theorem 5.12, we obtain that the md-spaces $\mathrm{X}\left(L / \theta_{Y}\right)$ and $Y$ are strong homeomorphic. From Proposition 7.6, we have that the md-spaces $\mathrm{X}\left(L / \theta_{Y}\right)$ and $X_{\theta_{Y}}$ are strong homeomorphic. Therefore $Y$ and $X_{\theta_{Y}}$ are strong homeomorphic and thus (7.3) holds.

Therefore, we have proved that the md-congruences on an md-semilattice $L$ are topologically represented by the md-subspaces of the dual md-space $\mathrm{X}(L)$ of $L$.

\section{REFERENCES}

[1] G. Bezhanishvili and R. Jansana. Duality for distributive and implicative semi-lattices. Preprints of University of Barcelona Research Group in Non-Classical Logics, 2008.

[2] G. Bezhanishvili and R. Jansana. Priestley style duality for distributive meet-semilattices. Studia Logica 98 (2011), no. 1-2, 83-122. MR 2821271

[3] S. Celani and I. Calomino. Stone style duality for distributive nearlattices. Algebra Universalis 71 (2014), no. 2, 127-153. MR 3183387.

[4] S. Celani and L. González. Notes on mildly distributive semilattices. Math. Slovaca 67 (2017), no. 5, 1073-1084. MR 3707112

[5] S. A Celani. Topological representation of distributive semilattices. Sci. Math. Jpn. 58 (2003), no. 1, 55-65. MR 1987817

[6] B. A. Davey and H. A. Priestley. Introduction to lattices and order. 2nd edition. Cambridge University Press, 2002. MR 1902334 
[7] E. David and M. Erné. Ideal completion and Stone representation of ideal-distributive ordered sets. Topology Appl. 44 (1992), no. 1-3, 95-113. MR 1173247

[8] R. Engelking. General topology, 2nd edition, Sigma Series in Pure Mathematics, vol. 6. Heldermann Verlag, 1989. MR 1039321

[9] O. Frink. Ideals in partially ordered sets. Amer. Math. Monthly 61 (1954), no. 4, 223-234. MR 0061575

[10] L. González and R. Jansana. A spectral-style duality for distributive posets. Order (2017). http://dx.doi.org/10.1007/s11083-017-9435-2

[11] G. Grätzer. Lattice theory: foundation. Birkhäuser, 2011. MR 2768581

[12] R. Hickman. Mildly distributive semilattices. J. Austral. Math. Soc. Ser. A 36 (1984), no. 3, 287-315. MR 0733904

[13] P. Johnstone. Stone spaces. Cambridge University Press, 1982. MR 0698074

[14] H. Priestley. Representation of distributive lattices by means of ordered Stone spaces. Bull. London Math. Soc. 2 (1970), 186-190. MR 0265242

[15] M. H. Stone. The theory of representations for Boolean algebras. Trans. Amer. Math. Soc. 40 (1936), no. 1, 37-111. MR 1501865 .

[16] M. H. Stone. Topological representations of distributive lattices and Brouwerian logics. Časopis pro pěstování matematiky a fysiky 67 (1938), issue 1, 1-25. Available at https: //dml.cz/handle/10338.dmlcz/124080

Sergio A. Celani ${ }^{凶}$

Universidad Nacional del Centro, Pinto 399, 7000 Tandil, Argentina

scelani@exa.unicen.edu.ar

Luciano J. González

Universidad Nacional de La Pampa, Uruguay 151, 6300 Santa Rosa, Argentina

lucianogonzalez@exactas.unlpam.edu.ar

Received: September 5, 2017

Accepted: February 13, 2018 